\title{
Detection of Gain Enhancement in Laser-Induced Fluorescence of Rhodamine B Lasing Dye by Silicon Dioxide Nanostructures-Coated Cavity
}

\author{
Mohammed N. A. AL-TAMEEMI* \\ Department of Physics, Al-Iraqia University, Baghdad, 12001, Iraq \\ *Corresponding author: Mohammed N. A. AL-TAMEEMIＩE-mail: mdnawfell@gmail.com
}

\begin{abstract}
In this work, nanostructured silicon dioxide films are deposited by closed-field unbalanced direct-current (DC) reactive magnetron sputtering technique on two sides of quartz cells containing rhodamine $\mathrm{B}$ dye dissolved in ethanol with $10^{-5} \mathrm{M}$ concentration as a random gain medium. The preparation conditions are optimized to prepare highly pure $\mathrm{SiO}_{2}$ nanostructures with a minimum particle size of about $20 \mathrm{~nm}$. The effect of $\mathrm{SiO}_{2}$ films as external cavity for the random gain medium is determined by the laser-induced fluorescence of this medium, and an increase of about $200 \%$ in intensity is observed after the deposition of nanostructured $\mathrm{SiO}_{2}$ thin films on two sides of the dye cell.
\end{abstract}

Keywords: Silicon dioxide; nanostructures; gain enhancement; rhodamine B dye

Citation: Mohammed N. A. AL-TAMEEMI, "Detection of Gain Enhancement in Laser-Induced Fluorescence of Rhodamine B Lasing Dye by Silicon Dioxide Nanostructures-Coated Cavity," Photonic Sensors, 2018, 8(1): 80-87.

\section{Introduction}

Scattering varies with the average radius of the particle, index of refraction, scatterers density, and the incident wavelength. If the particle size is small compared to the wavelength, Rayleigh scattering (in all directions) occurs, whereas bigger particles reflect and refract light $[1,2]$. If there are many scatterers randomly distributed inside the medium, the incident light wave will undergo scattering, and the scattered light will have a random path [3]. If the light waves have a long path inside the medium longer than the straight path of the waves, it will leave the medium without scattering. This is known as "multiple scattering", and it is not restricted to light waves but it's applied to all waves in nature [4-6]. There is an important parameter in multiple scattering; that is the mean free path $(l)$ which is defined as the average distance for a light wave transferred between two scattering events [6] and given by $[7,8]$

$$
l=\frac{1}{N_{\text {scatt }} \sigma_{\text {scatt }}} .
$$

where $N_{\text {scatt }}$ and $\sigma_{\text {scatt }}$ are the volume density and cross section of the scattering particles.

If the random amplification media have high scatterer' density, the mean free path of light will be short, and the interference effects of the scattered waves will be present alongside the gain of the active medium. The unification of interference and gain in a system results in a new laser output [9].

Improving the active medium of the dye laser has attracted the interest of many researches and studies. The first attempts started in the 1960 and

Received: 22 August 2017 / Revised: 25 September 2017

C The Author(s) 2017. This article is published with open access at Springerlink.com

DOI: $10.1007 / \mathrm{s} 13320-017-0462-9$

Article type: Regular 
continued up to now [10]. Rhodamine $\mathrm{B}(\mathrm{RB})$ dye is considered as a common typical material that belongs to the xanthene group and operates in the visible range [11]. The lasing emission of $\mathrm{RB}$ dye also depends on the type of solvent used for dissolving this dye to form its solution. For example, the central lasing wavelength is increased by about $43 \mathrm{~nm}$ when changing the solvent from ethanol to ethylene glycol. In general, the lasing emission is ranging within $570 \mathrm{~nm}-682 \mathrm{~nm}$ [12]. The unique properties of this dye have attracted the attention of researchers in several military, medical, and industrial applications The fluorescence lifetime of this dye is about $4.08 \times 10^{-9} \mathrm{~s}$, which means that the possibility of inter-system crossing is very weak $\left(<10^{7} \mathrm{~s}^{-1}\right)$ [13]. Using rhodamine dye as an active media of dye laser, it is possible to obtain continuous or pulsed laser output according to the pumping method in which a wide tunable wavelengths range can be obtained [14, 15].

Random laser has received much attention as a new development of laser physics and technology [16]. Its stimulated emission sources are different from those found in ordinary conventional lasers. The random laser is essentially built on insulating and semiconducting media [17]. It is characterized by being an "open resonator", i.e., it does not need an external optical cavity. Also, the feedback mechanism is provided using the so-called "scatterers" or "the tiny cavities" $[18,19]$. Random laser is characterized by its small size, simplicity, low cost, and applicability [20-22].

he interference between the scattered waves occurs when there is a high density of scatterers [23]. Since the transferring waves are exactly on the same distance, they maintain the same essential phase relationship even though the path is formed by a large number of scattering events [24, 25]. If the scattered waves are in phase with each other, constructive interference occurs and thus forming closed paths of light in the scattering media [26, 27]. These closed paths provide the coherent feedback mechanism [28-30]. The scatterers within the laser media contribute in trapping light photons due to multiple scattering; in doing so, the light path inside these media will be long (long time interval), and this is one reason for a decrease in the threshold intensity [31, 32].

In this work, nanostructured silicon dioxide films are deposited by closed-field unbalanced direct-current (DC) magnetron sputtering technique on two sides of quartz cells containing RB dye dissolved in ethanol with $10^{-5} \mathrm{M}$ concentration as a random gain medium. The preparation conditions are optimized to prepare highly pure $\mathrm{SiO}_{2}$ nanostructures with as minimum as possible particle size. The effect of $\mathrm{SiO}_{2}$ films as external cavity for the random gain medium is studied by the laser-induced fluorescence of this medium.

\section{Experiment}

A p-type silicon wafer of $10 \mathrm{~cm}$ in diameter and $300 \mu \mathrm{m}$ in thickness was used as the target to be sputtered and maintained carefully on the cathode. It was cleaned by hydrofluoric acid (HF), ethanol, and distilled water, dried, and then used for deposition process. Highly-pure argon and oxygen gases were used as discharge and reactive gases, respectively. The deposition process was performed on quartz cells. Before using them in sputtering experiments, these cells were first cleaned with ethanol to remove any oil layers or residuals may exist on their surfaces, which should be rinsed and washed with distilled water to remove ethanol, and then dried completely before being kept in clean case or placed inside the vacuum chamber. More details on the deposition system can be found in previous works $[33,34]$.

The RB laser dye solutions were prepared by dissolving the required amount of the dye in the ethanol. This amount of the dye $(W)$ was weighed using a precise digital balance of $10^{-4} \mathrm{~g}$ sensitivity (Matter Company) and could be calculated as [35] 


$$
W=\frac{M_{w} v c}{1000}
$$

where $M_{w}$ is the molecular weight of the dye ( $\mathrm{g} / \mathrm{mole}), v$ is the volume of the solvent $(\mathrm{ml})$, and $c$ is the molar concentration (mole/liter).

A high concentration of $10^{-2}$ mole/liter of RB dye solutions was prepared and then diluted to concentrations of $10^{-3} \mathrm{~mole} / \mathrm{liter}, 5 \times 10^{-3} \mathrm{~mole} / \mathrm{liter}$, $10^{-4}$ mole/liter, $5 \times 10^{-4}$ mole/liter, $10^{-5}$ mole/liter, $5 \times 10^{-5}$ mole/liter, and $10^{-6}$ mole/liter, respectively.

The operating conditions of the system were divided into two groups: constant and variable. The constant operating conditions include vacuum pressure, current limiting resistance, discharge voltage, discharge current, cooling temperature, cooling water flow rate, and deposition time. The variable operating conditions included gas pressure, inter-electrode distance, gas mixing ration, and gas flow rate. Varying discharge voltage was almost possible during the operation. In addition, turning the cooling system off would raise the temperature of either electrode to $40{ }^{\circ} \mathrm{C}-45^{\circ} \mathrm{C}$ with circulating water, while stopping the circulation of water would raise electrode temperature more (up to $150{ }^{\circ} \mathrm{C}$ ).

The quartz cell to be coated was completely immersed in the generated plasma. Big efforts were made to obtain uniform spatial distribution of plasma column in order to ensure homogeneous growth of prepared films. This could be done by controlling operation conditions and parameters, mainly the inter-electrode distance, gas flow, and discharge current.

The samples prepared in this work were characterized in order to determine their structural and spectral characteristics. The measurements and characterization tests include film thickness measurements, X-ray diffraction (XRD) patterns, atomic force microscopy (AFM), field-emission scanning electron microscopy (FE-SEM), energy-dispersive X-ray spectroscopy (EDS), Fourier-transform infrared spectroscopy (FTIR), ultraviolet-visible (UV-Vis) spectroscopy, and fluorescence measurements.

The X-ray diffraction patterns were recorded by a Shimadzu 6000 X-Ray Diffractometer system. This system measured the diffraction intensity as a function of Bragg's angle. The radiation source was $\mathrm{Cu}(\mathrm{k} \alpha)$ with wavelength of $1.5406 \AA$, current of $30 \mathrm{~mA}$, and voltage of $40 \mathrm{kV}$. The scanning angle $(2 \theta)$ could be varied in the range of 20 degree 60 degree with a speed of $4 \mathrm{deg} / \mathrm{min}$.

The SEM measurements were performed using MIRA3 Tescan FE-SEM instrument on samples prepared at the optimum conditions in order to introduce the prepared nanostructures as well as determine the nanoparticle size of the prepared samples. This instrument provided an EDS test to determine the content of elements in the final sample.

The FTIR measurements were carried out by FTIR spectrometer (SHIMADZU FTIR-8400S) on $\mathrm{KBr}$ pellets containing the prepared samples. These measurements were performed in the spectral range from $400 \mathrm{~cm}^{-1}$ to $4000 \mathrm{~cm}^{-1}$.

Transmission and absorption spectra of the prepared samples were recorded in the spectral range $200 \mathrm{~nm}-800 \mathrm{~nm}$ with an optical resolution of about $0.2 \mathrm{~nm}$ by using a computer-controlled UV-Vis spectrophotometer (K-MAC SpectraAcademy SV-2100). This measuring process could be precisely controlled by suitable software installed on a personal computer. The fluorescence spectra were recorded using F96 Fluorescence Spectrophotometer unit (Shanghai LengGuang Tech. Co., Ltd.) in the emission wavelengths range $180 \mathrm{~nm}-900 \mathrm{~nm}$, with xenon continuous-wave (CW) lamp as the excitation source.

In order to introduce the amplification activity in the prepared samples, the K-MAC SpectraAcademy SV-2100 instrument was used for amplified spontaneous emission (ASE) measurements after replacing the light source with a laser of $532 \mathrm{~nm}$ wavelength as an intensive light source required for the excitation of the prepared samples. The laser 
beam was incident on an empty face of the cell at $90^{\circ}$ with respect to the line between the coated faces and the photodetector.

The optimum conditions to prepare silicon dioxide nano films on the external walls of the quartz cell were inter-electrode distance of $4 \mathrm{~cm}$, Ar: $\mathrm{O}_{2}$ gas mixing ratio of 70:30, total gas pressure of 0.08 torr, discharge voltage of $2.5 \mathrm{kV}$, discharge current of $35 \mathrm{~mA}$, anode temperature of $150{ }^{\circ} \mathrm{C}$, and cathode temperature of $27^{\circ} \mathrm{C}$ (room temperature).

\section{Results and discussions}

Figure 1 shows the intensity of fluorescence spectra of RB dye dissolved in ethanol recorded at different concentrations $\left(10^{-3} \mathrm{M}, 10^{-4} \mathrm{M}, 10^{-5} \mathrm{M}\right.$, and $\left.10^{-6} \mathrm{M}\right)$. It is normal that decreasing the concentration of the dye solution leads to an increase in the fluorescence intensity. However, the difference between intensities for the concentrations of $10^{-5} \mathrm{M}$ and $10^{-6} \mathrm{M}$ are small compared with higher concentrations $\left(10^{-3} \mathrm{M}\right.$ and $\left.10^{-4} \mathrm{M}\right)$. These results are very useful to determine the concentration at which the highest fluorescence intensity is obtained.

The thickness of the prepared films is determined by laser interference fringes method to be $118 \mathrm{~nm}$ and $121 \mathrm{~nm}$ for two sides of the cubic quartz cells coated with $\mathrm{SiO}_{2}$ films after three hours deposition time.

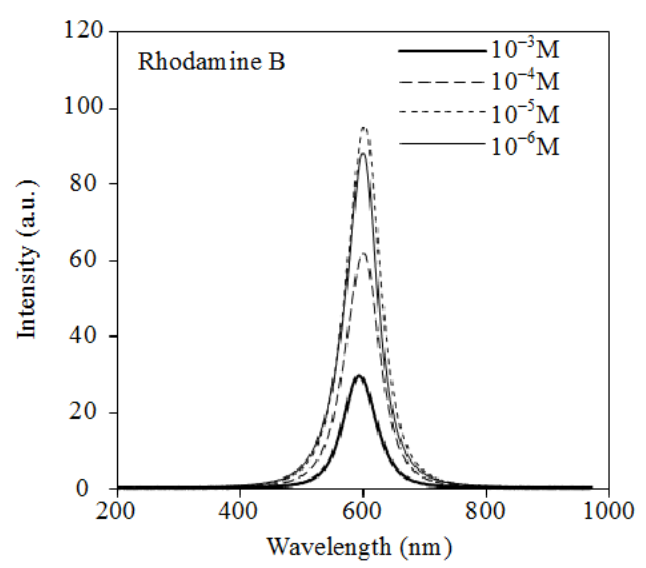

Fig. 1 Fluorescence spectra of RB dye dissolved in ethanol recorded at different concentrations.
Figure 2 shows the absorption and transmission spectra of the samples prepared at different deposition times ( $2 \mathrm{~h}, 2.5 \mathrm{~h}, 3 \mathrm{~h}, 3.5 \mathrm{~h}$, and $4 \mathrm{~h})$ in the spectral range $200 \mathrm{~nm}-800 \mathrm{~nm}$. It is clear that the sample prepared at shorter deposition time shows lower absorbance than those prepared at longer times. This is a typical behavior because a decrease in the deposition time leads to the growth of films with lower thickness as lower numbers of particle layers are grown on the substrate. Accordingly, the transmittance is decreased with increasing deposition time for the same reason.

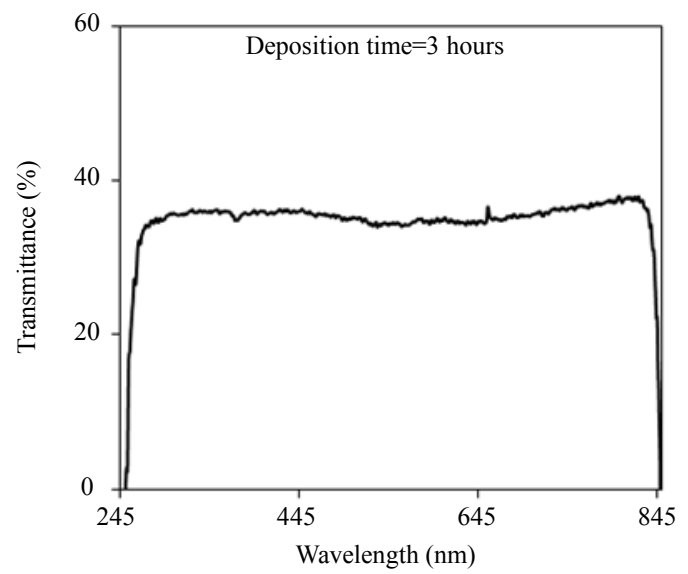

(a)

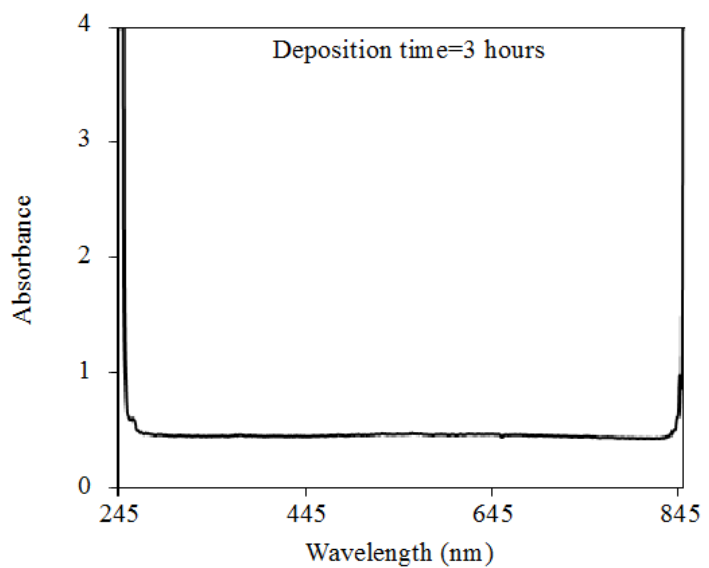

(b)

Fig. 2 Transmission (a) and absorption (b) spectra of the nanostructured $\mathrm{SiO}_{2}$ film prepared in this work after deposition time of 3 hours.

According to the observable stability of transmittance and absorbance values of the prepared 
samples over the spectral range of these measurements, these samples can be considered as optically homogeneous, and this is a very important feature for the applications to use such samples.

Figure 3 shows the XRD pattern of the $\mathrm{SiO}_{2}$ film sample prepared after deposition time of 3 hours. It is clearly observed that the prepared sample is amorphous, which is a characteristic of nanostructures; therefore, calculations of the structural parameters are not performed as no specific peak could be analyzed.

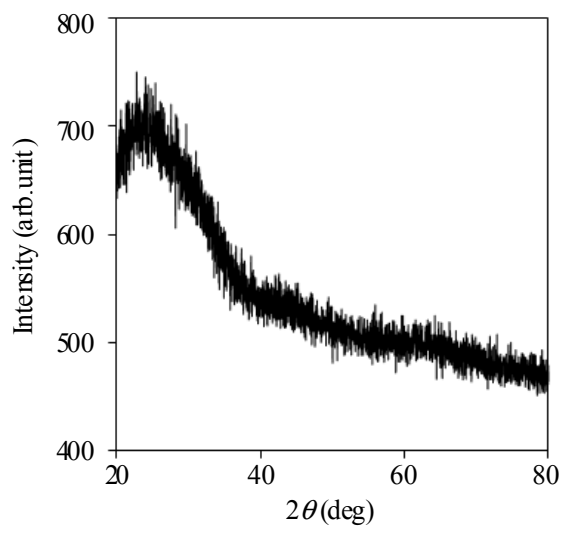

Fig. 3 XRD pattern of the nanostructured $\mathrm{SiO}_{2}$ film prepared in this work.

Figure 4 shows the SEM image for the $\mathrm{SiO}_{2}$ film sample prepared after deposition time of 3 hours. The minimum particle size for each sample is determined to be $20 \mathrm{~nm}$. The sample shows aggregation as large grains are formed.

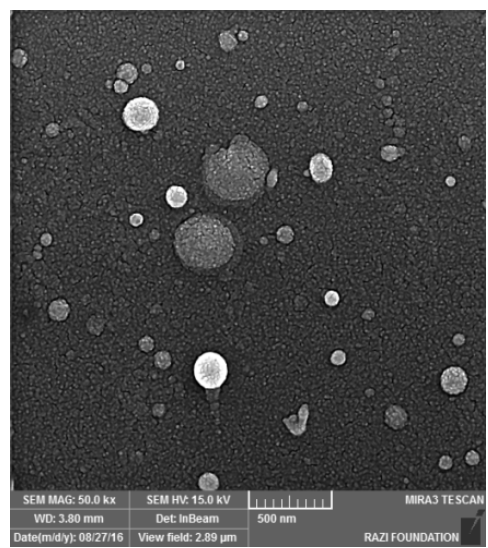

Fig. 4 SEM image of the nanostructured $\mathrm{SiO}_{2}$ film prepared in this work.

As the structural purity of the silicon dioxide $\left(\mathrm{SiO}_{2}\right)$ nanostructured thin films to be deposited on the dye cells in this work is highly required, the contents of composing elements (i.e., silicon and oxygen) in the final product are determined by the EDS measurements, as shown in Fig. 5. All samples show reasonably high peaks for both elements with lower peaks belonging to the elements which may be contained in the structure of the quartz substrates. However, the structures of these samples can be considered sufficiently pure. This structural purity is very important for these nanoparticles to play the role of scattering centers (scaterrers) for the random gain media. Weight percentage contents of silicon and oxygen in the final product are obtained from EDS results for the prepared samples to be $31.03 \mathrm{wt} . \%$ and $58.84 \mathrm{wt} . \%$, respectively.

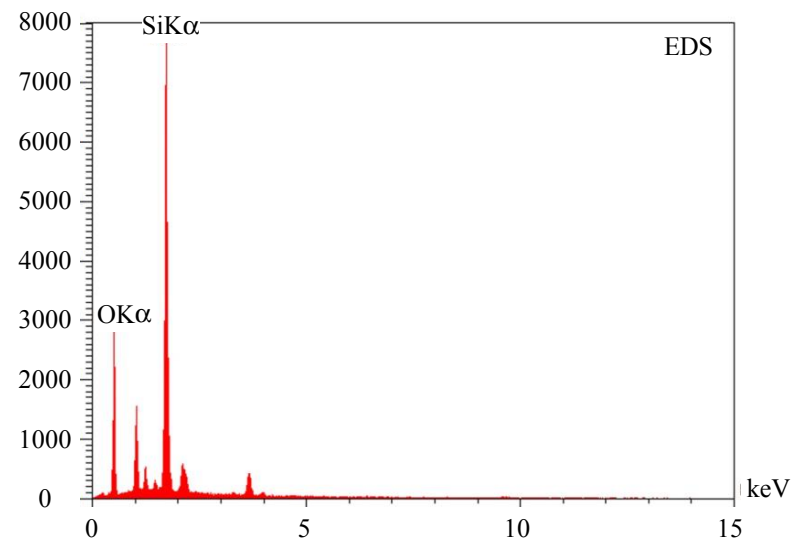

Fig. 5 EDS result of the nanostructured $\mathrm{SiO}_{2}$ film prepared in this work.

Figure 6 shows the 2D and 3D AFM images for the nanostructured $\mathrm{SiO}_{2}$ thin films deposited on the dye cells after deposition time of 3 hours. As can be seen, the aggregation on the surface of prepared sample is sufficiently low. The average diameter of particles is $91.83 \mathrm{~nm}$.

Certain applications of nanostructures, such as photodetectors, energy conversion devices, gas sensing, and ultra-hard coatings, require as high as possible surface area. Therefore, the prepared samples can be efficiently used for such applications.

In order to introduce the effect of nanostructured $\mathrm{SiO}_{2}$ thin films deposited on two sides of the dye 
cell on the fluorescence of the dye medium and hence on the gain characteristics, the fluorescence is recorded for the dye sample before and after the deposition of $\mathrm{SiO}_{2}$ films for $\mathrm{RB}$ dye, as shown in Fig. 7 at certain concentration of $10^{-5} \mathrm{M}$.

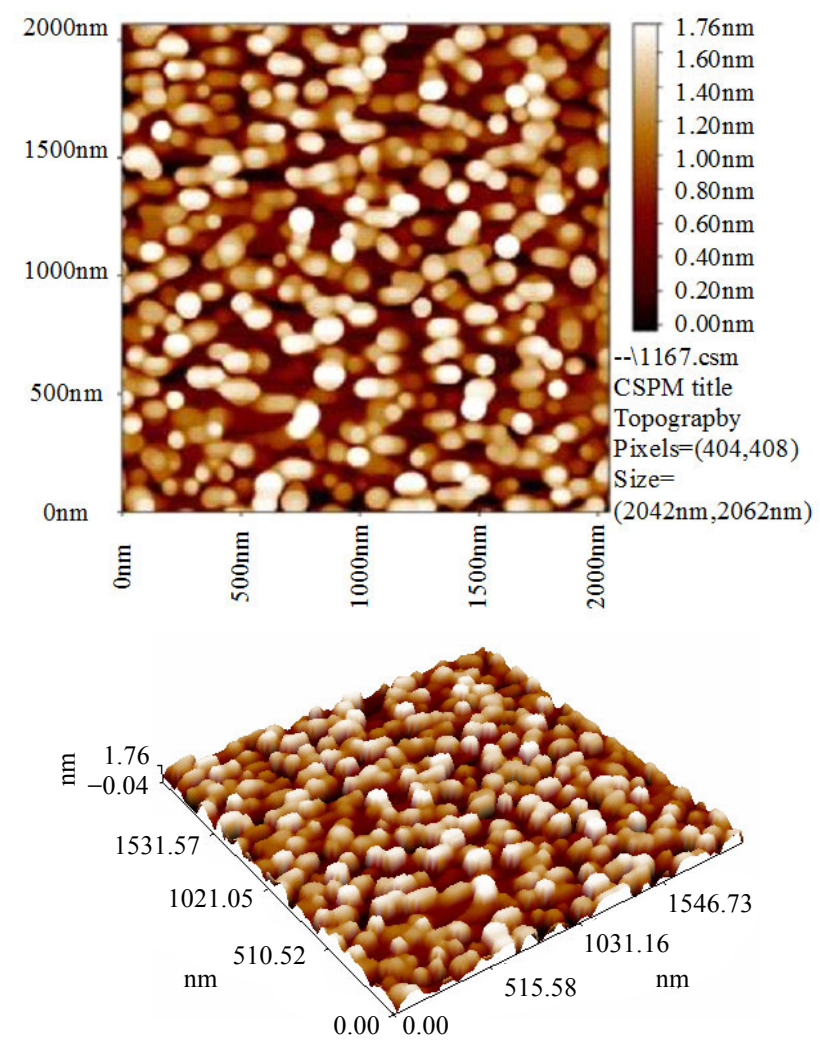

Fig. $62 \mathrm{D}$ and 3D AFM images for the nanostructured $\mathrm{SiO}_{2}$ film prepared in this work.

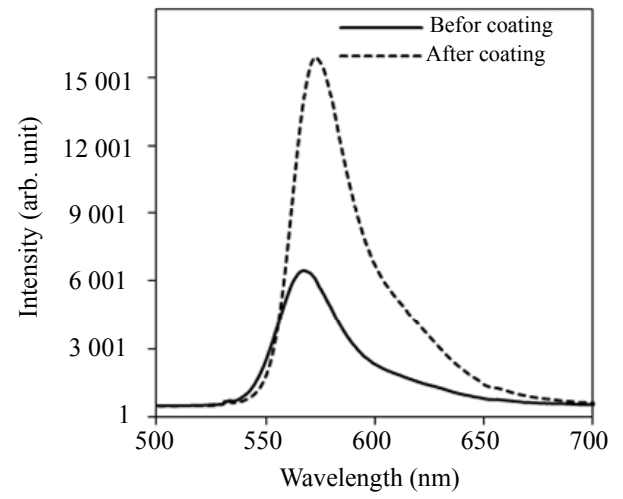

Fig. 7 Laser-induced fluorescence of $\mathrm{RB}$ dye before and after the deposition of nanostructured $\mathrm{SiO}_{2}$ thin films on two sides of the dye cell.

Light can be typically amplified in a random gain medium, and this amplification effect is proportional to the scattering mean free path of such medium as [36]

$$
l_{s}=\frac{1}{n_{s} \sigma_{s}}
$$

where $n_{s}$ is the number of scatterers, and $\sigma_{s}$ is the scattering cross-section of the individual scatterer.

Due to anisotropy in scattering, the scattering mean free path $\left(l_{s}\right)$ is not sufficient to describe this effect. Therefore, the average distance that the light travels before its direction of propagation is randomized is alternatively considered. This distance is known as transport mean free path $\left(l_{t}\right)$ defined by the following relation as [37]

$$
l_{t}=\frac{l_{s}}{1-g}
$$

where $g=<\cos \theta>$ is the average cosine of scattering angle $\theta$ (so-called the anisotropy parameter). For isotropic scattering (e.g., Rayleigh scattering from particle of size $<<\lambda), g=0$ or $l_{t}=l_{\mathrm{s}}$, hence $[38,39]$ :

$$
l_{t}=\frac{0.7 \times \lambda}{2 \pi w}
$$

where $\lambda$ is the excitation wavelength, and $W$ is the spectral scattering width of the ray reflected from the sample.

Nanostructures, in general, are described with very high surface roughness and hence very large number of single particles. Therefore, the value of $l_{s}$ is minimized according to (3). The transport mean free path $\left(l_{t}\right)$ is accordingly minimized, and the light is amplified within a very small distance as traversing through the random gain medium. The amplification effect is subsequently maximized. An increase of about $200 \%$ in laser-induced fluorescence intensity of RB dye is observed after the deposition of nanostructured $\mathrm{SiO}_{2}$ thin films on two sides of the dye cell.

\section{Conclusions}

In concluding remarks, highly-pure nanostructured silicon dioxide films with the minimum particle size of about $20 \mathrm{~nm}$ are deposited by the closed-field unbalanced dc magnetron sputtering technique on two sides of quartz cells 
containing RB dye dissolved in ethanol with $10^{-5} \mathrm{M}$ concentration to act as external cavity scatterers for this random gain medium. This role is determined by the laser-induced fluorescence of the dye sample, and an increase of about $200 \%$ in its intensity is observed after the deposition of nanostructured $\mathrm{SiO}_{2}$ thin films. These results can be successfully used to detect the gain enhancement in such media for spectroscopic and photonic applications.

Open Access This article is distributed under the terms of the Creative Commons Attribution 4.0 International License (http://creativecommons.org/ licenses/by/4.0/), which permits unrestricted use, distribution, and reproduction in any medium, provided you give appropriate credit to the original author(s) and the source, provide a link to the Creative Commons license, and indicate if changes were made.

\section{References}

[1] Y. Y. Chen and G. Jin, "Refractive index and thickness analysis of natural silicon dioxide film growing on silicon with variable-angle spectroscopic ellipsometry," Spectroscopy, 2006, 21(10): 26-31.

[2] A. Ranjgar, R. Norouzi, A. Zolanvari, and H. Sadeghi, "Characterization and optical absorption properties of plasmonic nanostructured thin films," Armenian Journal of Physics, 2013, 6(4): 198-203.

[3] O. A. Hamadi, "Characteristics of $\mathrm{CdO}-\mathrm{Si}$ heterostructure produced by plasma-induced bonding technique," Proceedings of the Institution of Mechanical Engineers Part L: Journal of Materials Design \& Applications, 2008, 222(L1): 65-71.

[4] A. Tabata, N. Matsuno, Y. Suzuoki, and T. Mizutani, "Optical properties and structure of $\mathrm{SiO}_{2}$ films prepared by ion-beam sputtering," Thin Solid Films, 1996, 289(1-2): 84-89.

[5] A. A. Anber and F. J. Kadhim, "Preparation of nanostructured $\mathrm{Si}_{x} \mathrm{~N}_{1-x}$ thin films by $\mathrm{DC}$ reactive magnetron sputtering for tribology applications," Silicon, 2017, 2017(2): 1-4.

[6] A. A. Issa, "The effect of annealing on nano-topography of $\mathrm{SiO}_{2}$ film," Rafidain Journal of Science, 2014, 25(2): 74-86.

[7] A. J. Haider, "The effect of some experimental parameters on the properties of porous silicon," Iraqi Journal of Applied Physics, 2008, 4(1): 37-40.

[8] S. E. Alexandrov, N. McSporran, and M. L. Hitchman, "Remote AP-PECVD of silicon dioxide films from hexamethyldisiloxane (HMDSO)," Chemical Vapor
Deposition, 2005, 11(11-12): 481-490.

[9] S. B. Bang, T. H. Chung, Y. Kim, M. S. Kang, and J. K. Kim, "Effects of the oxygen fraction and substrate bias power on the electrical and optical properties of silicon oxide films by plasma enhanced chemical vapour deposition using $\mathrm{TMOS} / \mathrm{O}_{2}$ gas," Journal of Physics D: Applied Physics, 2004, 37(12): 1679-1684.

[10] J. K. Choi, D. H. Kim, J. Lee, and J. B. Yoo, "Effects of process parameters on the growth of thick $\mathrm{SiO}_{2}$ using plasma enhanced chemical vapor deposition with hexamethyldisilazane," Surface \& Coatings Technology, 2000, 131(1): 136-140.

[11] D. Hiller, R. Zierold, J. Bachmann, M. Alexe, Y. Yang, J. W. Gerlach, et al., "Low temperature silicon dioxide by thermal atomic layer deposition: investigation of material properties," Journal of Applied Physics, 2010, 107(6): 064314-1 -064314-10.

[12] Y. Inoue and O. Takai, "Spectroscopic studies on preparation of silicon oxide films by PECVD using organosilicon compounds," Plasma Sources Science \& Technology, 1996, 5(2): 339-343.

[13] S. Kamiyama, T. Miura, and Y. Nara, "Comparison between $\mathrm{SiO}_{2}$ films deposited by atomic layer deposition with $\mathrm{SiH}_{2}\left[\mathrm{~N}\left(\mathrm{CH}_{3}\right)_{2}\right]_{2}$ and $\mathrm{SiH}\left[\mathrm{N}\left(\mathrm{CH}_{3}\right)_{2}\right]_{3}$ precursors," Thin Solid Films, 515(4): 1517-1521.

[14] J. W. Klaus and S. M. George, "Atomic layer deposition of $\mathrm{SiO}_{2}$ at room temperature using $\mathrm{NH}_{3}$-catalyzed sequential surface reactions," Surface Science, 2000, 447(1-3): 81-90.

[15] J. W. Klaus, A. W. Ott, J. M. Johnson, and S. M. George, "Atomic layer controlled growth of $\mathrm{SiO}_{2}$ films using binary reaction sequence chemistry," Applied Physics Letters, 1997, 70(9): 1092-1094.

[16] J. W. Klaus, O. Sneh, A. W. Ott, and S. M. George, "Atomic layer deposition of $\mathrm{SiO}_{2}$ using catalyzed and uncatalyzed selflimiting surface reactions," Surface Review \& Letters, 1999, 06(03n04): 435-448.

[17] J. H. Lee, C. H. Jeong, J. T. Lim, N. G. Jo, S. J. Kyung, and G. Y. Yeom, "Characteristic of $\mathrm{SiO}_{2}$ films deposited by using low temperature PECVD with TEOS $/ \mathrm{N}_{2} / \mathrm{O}_{2}$," Journal of the Korean Physical Society, 2005, 46: 890-894.

[18] J. H. Lee, U. J. Kim, C. H. Han, S. K. Rha, W. J. Lee, and C. O. Park, "Investigation of silicon oxide thin films prepared by atomic layer deposition using $\mathrm{SiH}_{2} \mathrm{Cl}_{2}$ and $\mathrm{O}_{3}$ as the precursors," Japanese Journal of Applied Physics, 2004, 43(3A): L328-L330.

[19] S. W. Lee, K. Park, B. Han, S. H. Son, S. K. Rha, C. O. Park, et al., "Atomic layer deposition of silicon oxide thin films by alternating exposures to $\mathrm{Si}_{2} \mathrm{Cl}_{6}$ and $\mathrm{O}_{3}, "$ Electrochemical and Solid-State Letters, 
2008, 11(7): G23-G26.

[20] M. P. Yu, H. Qiu, X. B. Chen, P. Wu, and Y. Tian, "Comparative study of the characteristics of Ni films deposited on $\mathrm{SiO}_{2} / \mathrm{Si}(100)$ by oblique-angle sputtering and conventional sputtering," Thin Solid Films, 2008, 516(21): 7903-7909.

[21] M. A. Hameed and Z. M. Jabbar, "Preparation and characterization of silicon dioxide nanostructures by DC reactive closed-field unbalanced magnetron sputtering," Iraqi Journal of Applied Physics, 2016, 12(4): 13-18.

[22] M. K. Dhahir and H. A. Khyoon, "Study the effect of $\mathrm{PH}$ variation on the particle size of $\mathrm{SiO}_{2}$ thin films," Iraqi Journal of Applied Physics, 2016, 15: 1-8.

[23] A. M. Mahajan, L. S. Patil, J. P. Bange, and D. K. Gautam, "Growth of $\mathrm{SiO}_{2}$ films by TEOS-PECVD system for microelectronics applications," Surface \& Coatings Technology, 2004, 183(2): 295-300.

[24] O. A. Hamadi, "Effect of annealing on the electrical characteristics of $\mathrm{CdO}-\mathrm{Si}$ heterostructure produced by plasma-induced bonding technique," Iraqi Journal of Applied Physics, 2008, 4(3): 34 -37 .

[25] O. A. Hammadi and N. E. Naji, "Electrical and spectral characterization of $\mathrm{CdS} / \mathrm{Si}$ heterojunction prepared by plasma-induced bonding," Optical \& Quantum Electronics, 2016, 48(8): 1-7.

[26] O. A. Hammadi, "Characteristics of heat-annealed silicon homojunction infrared photodetector fabricated by plasma-assisted technique," Photonic Sensors, 2016, 6(4): 345-350.

[27] O. A. Hammadi, "Characterization of $\mathrm{SiC} / \mathrm{Si}$ heterojunction fabricated by plasma-induced growth of nanostructured silicon carbide layer on silicon surface," Iraqi Journal of Applied Physics, 2016, 12(2): 9-13.

[28] O. A. Hammadi, "Photovoltaic properties of thermally-grown selenium-doped silicon photodiodes for infrared detection applications," Photonic Sensors, 2015, 5(2): 152-158.

[29] O. A. Hammadi, M. K. Khalaf, and F. J. Kadhim, "Fabrication and characterization of UV photodetectors based on silicon nitride nanostructures prepared by magnetron sputtering," Proceedings of the Institution of Mechanical Engineers Part N: Journal of Nanoengineering \& Nanosystems, 2015, 230(1): 32-36.
[30] O. A. Hammadi, M. K. Khalaf, and F. J. Kadhim, "Fabrication of UV photodetector from nickel oxide nanoparticles deposited on silicon substrate by closed-field unbalanced dual magnetron sputtering techniques," Optical \& Quantum Electronics, 2015, 47(12): 3805-3813.

[31] P. Pan, "The composition and properties of PECVD silicon oxide films," Applied Physics Letters, 1985, 132(8): 2012-2019.

[32] S. M. Zayed, A. M. Alshimy, and A. E. Fahmy, "Effect of surface treated silicon dioxide nanoparticles on some mechanical properties of maxillofacial silicone elastomer," International Journal of Biomaterials, 2014, 2014: 1-7.

[33] O. A. Hammadi, M. K. Khalaf, and F. J. Kadhim, "Silicon nitride nanostructures prepared by reactive sputtering using closed-field unbalanced dual magnetrons," Proceedings of the Institution of Mechanical Engineers Part L: Journal of Materials Design \& Applications, 2017, 231(5): 479-487.

[34] O. A. Hammadi, M. K. Khalaf, F. J. Kadhim, and B. T. Chiad, "Operation characteristics of a closed-field unbalanced dual-magnetrons plasma sputtering system," Bulgarian Journal of Physics, 2014, 41(1): 24-33.

[35] S. T. Sulaiman, Y. N. Al-Jammal, and A. A. Issa, "The growth and investigation of interface of $\mathrm{SiO}_{2} / \mathrm{Si}$ by anodic oxidation technique using acetic acid medium," Rafidain Journal of Science, 2012, 23(4): 117-126.

[36] I. Suzuki, C. Dussarrat, and K. Yanagita, "Extra low-temperature $\quad \mathrm{SiO}_{2} \quad$ deposition using aminosilanes," Ecs Transactions, 2007, 3(15): 119-128.

[37] T. Tamura, S. Ishibashi, S. Tanaka, M. Kohyama, and M. H. Lee, "First-principles analysis of optical absorption edge in pure and fluorine-doped $\mathrm{SiO}_{2}$ glass," Computational Materials Science, 2008, 44(1): 61-66.

[38] W. F. Wu and B. S. Chiou, "Optical and mechanical properties of reactively sputtered silicon dioxide films," Semiconductor Science \& Technology, 1996, 11(9): 1317-1321.

[39] Y. Chen and G. Jin, "Refractive index and thickness analysis of natural silicon dioxide film growing on silicon with variable-angle spectroscopic ellipsometry," Spectroscopy, 2006, 21(10): 26-31. 